\title{
DEFORMATIONS OF COMPLEX SUPERMANIFOLDS
}

\author{
MITCHELL J. ROTHSTEIN
}

\begin{abstract}
The supermanifold analogue of the Kodaira-Nirenberg-Spencer existence theorem for deformations of complex structures is given. It is shown that every complex supermanifold is a deformation of a vector bundle.
\end{abstract}

0. Supermanifolds, first used by physicists for modelling quantum gravity, have emerged as objects of independent interest. This paper will concentrate on supermanifolds with a complex structure, though the results also yield a simple and transparent proof of the fact that any supermanifold with only its $C^{\infty}$ structure is the sheaf of sections of a vector bundle [1].

Let $X$ be a complex manifold, with sheaf of holomorphic functions $\mathcal{O}$. Let $\mathscr{E}$ be a locally free sheaf of $\mathcal{O}$-modules. Then $\Lambda \mathscr{E}$, the sheaf of exterior algebras of $\mathscr{E}$ over $\mathcal{O}$, is an example of a complex supermanifold. $\Lambda \mathscr{E}$ is, among other things, a sheaf of supercommutative algebras. This means that $\Lambda \mathscr{E}$ is $\mathbf{Z}_{2}$-graded and $a b=(-1)^{|b||a|} b a$ for $a$ and $b$ of definite parity. $\Lambda \mathscr{E}$ is also a sheaf of $\mathcal{O}$-modules, and a sheaf of $\mathbf{Z}$-graded algebras, but for supersymmetry one is concerned only with the $\mathbf{Z}_{2}$-grading. This leads to the following more general definition:

Definition. A complex supermanifold of dimension $(m, n)$ is a sheaf $(M, \mathscr{A})$ of supercommutative algebras over $\mathbf{C}$ such that

(1) $(M, \mathscr{A} / \mathscr{N})$ is an $m$-dimensional complex manifold. $(\mathscr{N}$ is the ideal of nilpotent elements of $\mathscr{A}$.)

(2) The sheaves $(M, \mathscr{A})$ and $\left(M, \Lambda \mathbf{C}^{n} \otimes \mathscr{A} / \mathscr{N}\right)$ are locally isomorphic as sheaves of $\mathbf{Z}_{2}$-graded commutative algebras over $\mathbf{C}$.

Set $\mathcal{O}=\mathscr{A} / \mathscr{N}$ and $\mathscr{E}=\mathscr{N} / \mathscr{N}^{2}$. Then $\mathscr{E}$ is an $\mathcal{O}$-module, and it follows from (2) that $\mathscr{E}$ is locally free. That is, $\mathscr{E}$ is the sheaf of sections of a holomorphic vector bundle. By writing $\mathscr{A} / \mathscr{N}^{2}$ as the direct sum of its even and odd parts, one obtains an exact sequence of sheaves of vector spaces

$$
0 \rightarrow \mathscr{N}^{2} \rightarrow \mathscr{A} \rightarrow \mathcal{O} \oplus \mathscr{E} \rightarrow 0
$$

If there is a splitting

$$
0 \rightarrow \mathcal{O}+\stackrel{\mu}{\mathscr{\mu}} \rightarrow \mathscr{A}
$$

Received by the editors January 20, 1984 and, in revised form, December 27, 1984.

1980 Mathematics Subject Classification. Primary 32C35. 
of $(*)$ such that $\mu(f \xi)=\mu(f) \mu(\xi)$ for all $f \in \mathcal{O}$ and $\xi \in \mathcal{O} \oplus \mathscr{E}$, then $\mu$ extends to an isomorphism $\Lambda \mathscr{E} \simeq \mathscr{A}$. On the other hand, for the sequence

$$
0 \rightarrow \sum_{i \geqslant 2} \Lambda^{i} \mathscr{E} \rightarrow \Lambda \mathscr{E} \rightarrow \mathcal{O} \oplus \mathscr{E} \rightarrow 0,
$$

such a splitting surely exists. Thus if $\mathscr{A} \simeq \Lambda \mathscr{E}$, we say that $\mathscr{A}$ is split, and if not we say that $\mathscr{A}$ is nonsplit.

The idea will be to regard a nonsplit supermanifold as a deformation of a split one. We define the notion of an analytic family of supermanifolds, and show that to any supermanifold $(M, \mathscr{A})$ there is associated an analytic one parameter family of supermanifolds $(M, \mathscr{A}(z)), z \in \mathbf{C}$, such that $\mathscr{A}(0)=\Lambda \mathscr{E}$ and $\mathscr{A}(1)=\mathscr{A}$. We also attach to $\mathscr{A}$ an integer $n(\mathscr{A})$ and an invariant $\Delta \mathscr{A}$, which measure the failure of $\mathscr{A}$ to split. Finally, we prove that under suitable conditions it is possible to construct a supermanifold with a prescribed invariant.

1. Let $\mathscr{A}$ ut $\Lambda \mathscr{E}$ denote the sheaf of parity preserving $\mathbf{C}$-linear algebra automorphisms of $\Lambda \mathscr{E}$. Define $\Lambda^{(k)} \mathscr{E}=\sum_{j \geqslant k} \Lambda^{j} \mathscr{E}$. If $g: \Lambda \mathscr{E} \rightarrow \Lambda \mathscr{E}$ is an automorphism, then $g$ induces an $\mathscr{O}$-linear automorphism $g^{\prime}: \mathscr{E} \rightarrow \mathscr{E}$, by virtue of the identification $\mathscr{E} \simeq$ $\Lambda^{(1)} \mathscr{E} / \Lambda^{(2)} \mathscr{E}$. Denote by $\mathscr{A} u t^{+} \Lambda \mathscr{E}$ the subsheaf of automorphisms for which $g^{\prime}=i d$.

For $k$ an even integer, let $\mathscr{D} e r_{k} \Lambda \mathscr{E}$ denote the sheaf of derivations which increase degree by $k$. Let $\mathscr{D} e r^{(j)} \Lambda \mathscr{E}=\sum_{j \leqslant 2 k \leqslant n} \mathscr{D}_{e r_{2 k}} \Lambda \mathscr{E}$. For $Y \in \mathscr{D}_{e r^{(0)}} \Lambda \mathscr{E}$, let $Y_{k}$ denote the $\mathscr{D}_{e r_{k}} \Lambda \mathscr{E}$ component of $Y$. Explicitly, if $z^{1}, \ldots, z^{m}$ are coordinates on $M$ and $\pi^{1}, \ldots, \pi^{n}$ are a basis for $\mathscr{E}$ over $\mathcal{O}$, then any derivation is uniquely expressible in the form

$$
Y=\sum f^{i} \frac{\partial}{\partial z^{i}}+g^{j} \frac{\partial}{\partial \pi^{j}},
$$

where $f^{i}$ and $g^{j}$ are sections of $\Lambda \mathscr{E}$. Then $Y$ lies in $\mathscr{D}_{e r_{k}} \Lambda \mathscr{E}$ if and only if, for all $i$ and $j, \operatorname{deg}\left(f^{i}\right)=k$ and $\operatorname{deg}\left(g^{j}\right)=k+1$.

Elements of $\mathscr{D} e \imath^{(2)} \Lambda \mathscr{E}$ are nilpotent, so the power series exp: $\mathscr{D} e \imath^{(2)} \Lambda \mathscr{E} \rightarrow \mathscr{A} u t \Lambda \mathscr{E}$ is well defined.

Proposition 1. exp: $\mathscr{D} e r^{(2)} \Lambda \mathscr{E} \rightarrow \mathscr{A} u t^{+} \Lambda \mathscr{E}$ is bijective.

PROOF. For $Y \in \mathscr{D} e r^{(2)} \Lambda \mathscr{E}$, it is clear that $\exp Y$ induces the identity on $\mathscr{E}$. On the other hand, for $g \in \mathscr{A} u t^{+} \Lambda \mathscr{E}, 1-g$ is degree increasing and therefore nilpotent, so $\log g$ is well defined and lies in $\mathscr{D} e^{(2)} \Lambda \mathscr{E}$.

Define the order of $\mathscr{A}$, denoted $o(\mathscr{A})$, as the sup of integers $k \leqslant n+1$ such that $\mathscr{A} / \mathscr{N}^{k}$ and $\Lambda \mathscr{E} / \Lambda^{(k)} \mathscr{E}$ are isomorphic. Assuming $n \geqslant 1, o(\mathscr{A})$ is at least 2 , and is either even or equal to $n+1$.

Let $\Xi$ be an open cover of $M$ such that, for all $u \in \Xi$, the isomorphism (2) of the definition exists and $\mathscr{E}$ is trivial over $u$. $\mathscr{A}$ is filtered by $\mathscr{N}$, and the associated graded sheaf is $\Lambda \mathscr{E}$. For each $u \in \Xi$, an isomorphism $T_{u}:\left.\left.\mathscr{A}\right|_{u} \rightarrow \Lambda \mathscr{E}\right|_{u}$ can be chosen in such a way that the associated map of $\mathbf{Z}$-graded algebras is the identity. The cocycle $\left\{T_{u} T_{v}^{-1} \mid u, v \in \Xi\right\}$ defines $\mathscr{A}$ up to isomorphism, and it follows that the isomorphism classes of supermanifolds $(X, \mathscr{A})$ with underlying $\mathcal{O}$-module $\mathscr{E}$ are in natural 1-1 correspondence with $H^{1}\left(M, \mathscr{A} u t^{+} \Lambda \mathscr{E}\right)$. 
Proposition 2. There exists a cocycle $\exp \left(Y^{u v}\right)$ defining $\mathscr{A}$ such that, for all $u, v \in \Xi$ and all $j<o(\mathscr{A}), Y_{j}^{u v}=0$.

Proof. Observe that any automorphism of $\Lambda \mathscr{E} / \Lambda^{(k)} \mathscr{E}$ is determined by its restriction to $\mathcal{O}+\mathscr{E}$. It follows that, for all $u \in \Xi$, the natural map $\mathscr{A} u t^{+} \Lambda \mathscr{E}(u) \rightarrow$ $\mathscr{A} u t^{+}\left(\Lambda \mathscr{E} / \Lambda^{(k)} \mathscr{E}\right)(u)$ is surjective. Denote this map by $\pi$. Now choose a cocycle $\exp \left(Z^{u v}\right)$ defining $\mathscr{A}$. Then the cocycle $\pi \exp \left(Z^{u v}\right)$ defines $\mathscr{A} / \mathscr{N}^{k}$. If $\mathscr{A} / \mathscr{N}^{k}$ and $\Lambda \mathscr{E} / \Lambda^{(k)} \mathscr{E}$ are isomorphic, there exist automorphisms $\rho^{u} \in \mathscr{A} u t^{+}\left(\Lambda \mathscr{E} / \Lambda^{(k)} \mathscr{E}\right)(u)$ such that

$$
\pi \exp \left(Z^{u v}\right)=\rho^{u}\left(\rho^{v}\right)^{-1}
$$

$\rho^{u}$ is of the form $\pi \exp \left(X^{u}\right)$, for some $X^{u} \in \mathscr{D} e r^{(2)} \Lambda \mathscr{E}(u)$. Then the cocycle

$$
\exp \left(Y^{u v}\right)=\exp \left(-X^{u}\right) \exp \left(Z^{u v}\right) \exp \left(X^{v}\right)
$$

defines $\mathscr{A}$ and satisfies $Y_{j}^{u v}=0$ for $j \leqslant k$, as desired.

Let $\tau=\exp \left(Y^{u v}\right)$ be a cocycle with coefficients in $\mathscr{A} u t^{+} \Lambda \mathscr{E}$. Call $\tau$ reduced if $\tau$ satisfies the property in Proposition 2.

For all $u, v$ and $w \in \Xi$,

$$
\exp \left(Y^{u w}\right)=\exp \left(Y^{u v}\right) \exp \left(Y^{v w}\right)=\exp \left(Y^{u v}+Y^{v w}+\text { commutator terms }\right) \text {. }
$$

So if $\tau$ is reduced, then, for $j<2 o(\mathscr{A}), Y_{j}$ is an additive cocycle. Thus $Y$ determines a class $\omega(\tau) \in H^{1}\left(M, \mathscr{D} e r^{(o(\mathscr{A}))} \Lambda \mathscr{E} / \mathscr{D} e r^{(2 o(\mathscr{A}))} \Lambda \mathscr{E}\right)$.

Denote the group $H^{0}\left(M, \mathscr{A} u t^{+} \Lambda \mathscr{E}\right)$ of global sections of $\mathscr{A} u t^{+} \Lambda \mathscr{E}$ by $G^{+}(\Lambda \mathscr{E})$. $G^{+}(\Lambda \mathscr{E})$ acts on $\mathscr{D}_{e r^{(o(\mathscr{A}))}} \Lambda \mathscr{E} / \mathscr{D}_{e} r^{(2 o(\mathscr{A}))} \Lambda \mathscr{E}$ by conjugation, and one has

Proposition 3. The orbit of $\omega(\tau)$ under the action of $G^{+}(\Lambda \mathscr{E})$ is an invariant of $(M, \mathscr{A})$.

Proof. Let $\sigma=\exp \left(X^{u v}\right)$ be another cocycle defining $\mathscr{A}$. Then there is a 0 -cochain $Z^{u}$ such that $\exp \left(X^{u v}\right)=\exp \left(Z^{u}\right) \exp \left(Y^{u v}\right) \exp \left(-Z^{v}\right)$. If $X_{j}^{u v}=0$ for all $j<o(\mathscr{A})$, then it follows by induction on $j$ that $Z_{j}^{u}=Z_{j}^{v}$ for all $u$ and $v \in \Xi$ and all $j<o(\mathscr{A})$. For $j \geqslant o(\mathscr{A})$ and $k<2 o(\mathscr{A})$, the $Z_{j}^{u}$ terms have no effect on the cohomology class of $Y_{k}$. Thus $\omega(\sigma)$ and $\omega(\tau)$ are conjugate under $\exp (Z)$, where $Z$ is defined by $\left.Z\right|_{u}=\sum_{2 \leqslant j<o(\mathscr{A})} Z_{j}^{u}$.

Definition. Let $\Delta \mathscr{A}$ denote the orbit of $\omega(\tau)$ under $G^{+}(\Lambda \mathscr{E})$.

THEOREM 1. A splits if and only if $\Delta \mathscr{A}=0$.

Proof. Let $\tau=\exp (Y)$ be a reduced cocycle representing $\mathscr{A}$. If $\mathscr{A}$ splits, then $\tau=1$, so that $\omega(\tau)=0$. On the other hand, assume $\omega(\tau)=0$. Then there is a 0 -cochain $Z$ with coefficients in $\mathscr{D}_{e r_{o(\mathscr{A})}} \Lambda \mathscr{E}$ such that $Y_{o(\mathscr{A})}^{u v}=Z^{u}-Z^{v}$. Set $\sigma^{u v}=$ $\exp \left(-Z^{u}\right) \tau^{u v} \exp \left(Z^{v}\right)$. Then $\sigma=\exp (W)$ for some 1-cochain $W, \sigma$ defines $\mathscr{A}$, and $W_{j}^{u v}=0$ for $j \leqslant o(\mathscr{A})$. Unless $o(\mathscr{A})=n+1$, this is a contradiction.

2. A map $\Phi:(M, \mathscr{A}) \rightarrow(N, \mathscr{B})$ between two complex supermanifolds is a holomorphic map $\phi: M \rightarrow N$ together with a sheaf morphism $\phi^{\prime}: \phi^{-1} \mathscr{B} \rightarrow \mathscr{A}$. The notions of tangent space and differential map carry over directly to supermanifolds. For $p \in M, \Phi$ is called submersive at $p$ if $d \Phi_{p}$ is surjective. If $\Phi$ is submersive and if 
the dimensions of $(M, \mathscr{A})$ and $(N, \mathscr{B})$ are $\left(m_{0}, m_{1}\right)$ and $\left(n_{0}, n_{1}\right)$, respectively, then we must have $n_{i} \leqslant m_{i}$ for $i=0,1$. Moreover, $\phi$ is submersive. (See [6 and 7] for general background.)

Assume $\Phi$ is everywhere submersive. If the odd dimension of $\mathscr{A}$ is 0 , then for all $q \in N$, the structure sheaf of the fiber over $q$ is the quotient of $\left.\mathcal{O}_{M}\right|_{\phi^{-1} q}$ by $\mathscr{I}_{q}$, where $\mathscr{I}_{q}$ is the ideal whose members vanish along $\phi^{-1}(q)$. In general, however, the nilpotent elements of $\mathscr{A}$ take the value 0 at all points of $M$, and one does not want the fiber supermanifolds to be devoid of nilpotents. So the procedure for constructing $\mathscr{I}_{q}$ in general is to start by taking a derivation $\left.X \in \mathscr{D} e r \mathscr{A}\right|_{p}$ and saying $X$ is vertical if $X$ annihilates $\left.\phi^{\prime}\left(\phi^{-1} \mathscr{B}\right)\right|_{p}$. Then let $\delta$ denote the quotient map

$$
0 \rightarrow \mathscr{N} \rightarrow \mathscr{A} \stackrel{\delta}{\rightarrow} \mathcal{O} \rightarrow 0 .
$$

Finally, say $f$ is in $\left.\mathscr{I}_{q}\right|_{p}$ if and only if for all nonnegative integers $k$ and all vertical derivations $X_{1}, \ldots, X_{k}$ at $p, \delta \circ X_{1} \circ \ldots \circ X_{k} f$ vanishes along $\phi^{-1}(q)$.

An explicit description of $\mathscr{I}_{q}$ in terms of local coordinates adapted to $\Phi$ reveals that $\left.\mathscr{A}\right|_{\phi^{-1} q} / \mathscr{I}_{q}$ is a supermanifold over $\phi^{-1} q$ with dimension $\left(m_{0}-n_{0}, m_{1}-n_{1}\right)$. Thus $(M, \mathscr{A})$ fibers over $(N, \mathscr{B})$, and we call $(M, \mathscr{A})$ an analytic family of supermanifolds parameterized by $(N, \mathscr{B})$.

As an example of such a family, consider the action of $\mathbf{C}$ on $\mathscr{D} e r^{(0)} \Lambda \mathscr{E}$, given by $z \cdot Y=z^{j} Y, Y \in \mathscr{D}_{e r_{2 j}} \Lambda \mathscr{E}$. Then $\mathbf{C}$ acts by homomorphisms on $\mathscr{A} u t^{+} \Lambda \mathscr{E}$, by $z \exp (Y)=\exp (z \cdot Y)$. This descends to an action of $\mathbf{C}$ on

$$
H^{1}\left(M, \mathscr{D} e r^{(k)} \Lambda \mathscr{E} / \mathscr{D} e r^{(l)} \Lambda \mathscr{E}\right) / G^{+} \Lambda \mathscr{E}, \quad k<l .
$$

THEOREM 2. Let $(M, \mathscr{A})$ be a supermanifold defined by some $\tau \in$ $H^{1}\left(M, \mathscr{A} u t^{+} \Lambda \mathscr{E}\right)$. Then the classes $z \cdot \tau, z \in \mathbf{C}$, determine an analytic family of supermanifolds parameterized by $\mathbf{C}$. If we denote the supermanifold on the zth fiber by $\mathscr{A}(z)$, then $\Delta \mathscr{A}(z)=z \cdot \Delta \mathscr{A}$.

Proof. Represent $\tau$ by a cocycle $\exp \left(Y^{u v}\right)$ defined on an open cover $\Xi$ of $M$. The $\mathcal{O}_{M}$ module $\mathscr{E}$ pulls back to an $\mathcal{O}_{M \times \mathbf{C}}$ module $\mathscr{E}^{\prime}$ on $M \times \mathbf{C}$, and derivations of $\Lambda \mathscr{E}$ act on $\Lambda \mathscr{E}^{\prime}$ by ignoring the $z$ coordinate. Thus $z \cdot \tau$ can be regarded as lying in $H^{1}\left(M \times \mathbf{C}, \mathscr{A} u t^{+} \Lambda \mathscr{E}^{\prime}\right)$, and so determines a supermanifold $\mathscr{B}$ on $M \times \mathbf{C}$. The projection $\pi: M \times \mathbf{C} \rightarrow \mathbf{C}$ induces an injection $\pi^{*}: \pi^{-1}\left(\mathcal{O}_{\mathbf{C}}\right) \rightarrow \mathcal{O}_{M \times \mathbf{C}} \rightarrow \Lambda \mathscr{E}^{\prime}$ on whose image the automorphisms $\exp \left(z Y^{u v}\right)$ act trivially. Thus $\pi^{*}$ induces an injection $\pi^{\prime}: \pi^{-1}\left(\mathcal{O}_{\mathbf{C}}\right) \rightarrow \mathscr{B}$ making $(M \times \mathbf{C}, \mathscr{B})$ an analytic family. That the fibers have the desired invariant is a direct verification.

The classical "no obstruction" theorem for deformations of complex structures [5] carries over to the supermanifold case.

Proposition 4. Let $\mathrm{g}=\sum_{i \geqslant 1} \mathfrak{g}_{i}$ be a sheaf of Z-graded Lie algebras over a space $M$. Fix positive integers $j$ and $k$, with $j \leqslant k$. Let $Y$ be a 1-cochain with coefficients in $\sum_{j \leqslant i \leqslant k} \mathfrak{g}_{i}$. Set $\exp \left(Y^{u v}\right) \exp \left(Y^{v w}\right)=\exp \left(Y^{u w}+Z^{u v w}\right)$, and assume that the 2-cochain $Z$ has coefficients in $\sum_{i \geqslant(k+1)} \mathfrak{g}_{i}$. Then $Z_{k+1}$ is a cocycle. 
Proof. This is proved by induction on $k-j$. Note that $Y_{j}$ is a cocycle. If $k=j$, then $Z^{u v w}$ is the $(k+1)$ st component of $\frac{1}{2}\left[Y^{u v}, Y^{v w}\right]$, which is easily seen to be a cocycle. Since the proposition is local, we may restrict our attention to a neighborhood on which $Y_{j}^{u v}=X^{u}-X^{v}$ for some 0 -chain $X$. If we replace $Y$ by $Y^{\prime}$, where $Y^{\prime}$ is defined by

$$
\exp \left(Y^{\prime u v^{\prime}}\right)=\exp \left(-X^{u}\right) \exp \left(Y^{u v^{\prime}}\right) \exp \left(X^{v}\right)
$$

then the $(k+1)$ st component of $Z$ is unchanged, whereas $Y_{j}^{\prime}=0$. Thus the induction proceeds.

From this follows

THEOREM 3. Let $k$ be an even integer, $k \geqslant 2$. Let $V$ be a finite-dimensional subspace of $H^{1}\left(M, \mathscr{D}_{e} r^{(k)} \Lambda \mathscr{E} / \mathscr{D}_{e} r^{(2 k)} \Lambda \mathscr{E}\right)$. Assume $H^{2}\left(M, \mathscr{D}_{e} r^{(2 k)} \Lambda \mathscr{E}\right)=0$. Then there is an analytic family of supermanifolds parameterized by $V$ such that, for all $\omega \in V$,

$$
\Delta \mathscr{A}(\omega)=G^{+}(\Lambda \mathscr{E}) \cdot \omega \text {. }
$$

Proof. Fix a basis $\omega_{1}, \ldots, \omega_{r}$ for $V$. For $i=1, \ldots, r$, let $Y_{j}$ be a cocycle representing $\omega_{j}$. Now consider the sheaf of Z Z-graded Lie algebras $\mathfrak{g}=\mathbf{C}\left[z^{1}, \ldots, z^{r}\right] \otimes$ $\mathscr{D} e r^{(k)} \Lambda \mathscr{E}$, with grading inherited from the second factor. To prove the theorem we must find a 1-cochain $Y$ with coefficients in $g$ such that

(i) $Y \equiv \sum z^{j} \otimes Y_{j}$ modulo $\mathscr{D} e r^{(2 k)} \Lambda \mathscr{E}$, and

(ii) $\exp (Y)$ is a cocycle.

Proposition 4 guarantees that the obstruction to finding this cochain lies in $H^{2}\left(M, \mathscr{D} e r^{(2 k)} \Lambda \mathscr{E}\right)$, which vanishes. Note that the Z-grading on $\mathscr{D} e r^{(k)} \Lambda \mathscr{E}$ allows for a polynomial dependence on $V$, and thereby circumvents the problem of convergence.

3. The same considerations apply to $C^{\infty}$ supermanifolds. In that case, $\mathscr{D} e r^{(0)} \Lambda \mathscr{E}$ is a fine sheaf, so $\Delta \mathscr{A}=0$. Therefore, Theorem 1 yields the theorem of Batchelor that all smooth supermanifolds are in fact vector bundles [1]. Also see [3] for the first proof of this result.

4. The conditions in Theorem 3 are easily achieved. For example, if $k>n / 2$, then the hypothesis $H^{2}\left(M, \mathscr{D}_{e r^{(2 k)}} \Lambda \mathscr{E}\right)=0$ is vacuous. In fact, the ideal $\mathscr{D}_{e} r^{(k)} \Lambda \mathscr{E}$ is abelian, so any class $\omega \in H^{1}\left(M, \mathscr{D}_{e} r^{(k)} \Lambda \mathscr{E}\right)$ can be exponentiated immediately to determine an isomorphism class of supermanifolds $\exp (\omega) \in H^{1}\left(M, \mathscr{A} u t^{+} \Lambda \mathscr{E}\right)$. This is the sort of example given in [4]. As a further example, suppose $\mathscr{E}$ is free and has rank $n$. Then

$$
\mathscr{D} e r^{(0)} \Lambda \mathscr{E}=\Lambda^{\text {even }} \mathscr{E} \otimes \Theta+\Lambda^{\text {odd }} \mathscr{E} \otimes \mathscr{E}^{*}=\Theta^{r}+\mathcal{O}^{s},
$$

where $r=2^{n-1}$ and $s=n 2^{n-1}$ and where $\Theta$ is the sheaf of holomorphic vector fields. So if $H^{1}(M, \Theta+\mathcal{O}) \neq 0$ and $H^{2}(M, \Theta+\mathcal{O})=0$, for instance if $M$ is a Riemann surface of positive genus, then $M$ carries supermanifolds of any order.

\section{REFERENCES}

1. M. Batchelor, The structure of supermanifolds, Trans. Amer. Math. Soc. 253 (1979), 329-338.

2. F. A. Berezin and D. A. Leites, Supermanifolds, Soviet Math. Dokl. 16 (1975), 1218-1221.

3. K. Gawedzki, Supersymmetries-Mathematics of supergeometry, Ann. Inst. H. Poincaré Sect. A (N.S.) 27 (1977), 335-366. 
4. P. Green, On holomorphic graded manifolds, Proc. Amer. Math. Soc. 85 (1982), 587-590.

5. K. Kodaira, L. Nirenberg and D. C. Spencer, On the existence of deformations of complex analytic structures, Ann. of Math. 68 (1958), 450-459.

6. B. Kostant, Graded manifolds, graded Lie theory, and prequantization, Differential Geometric Methods in Mathematical Physics (Proc. Sympos. Univ. Bonn, Bonn, 1975), Lecture Notes in Math., vol. 570, Springer-Verlag, Berlin, 1977, pp. 177-306.

7. D. A. Leites, Introduction to the theory of supermanifolds, Russian Math. Surveys 35 (1980), 1-64.

8. I. B. Penkov, D-modules on supermanifolds, Invent. Math. 71 (1983), 501-512.

9. A. Salam and J. Strathdee, Superfields and Fermi-Bose symmetry, Phys. Rev. D (3) 11 (1975), 1521-1535.

Department of Mathematics, University of Washington, Seattle, Washington 98195 